\title{
REMOCIÓN DE PLOMO Y NÍQUEL EN SOLUCIONES ACUOSAS USANDO BIOMASAS LIGNOCELULÓSICAS: UNA REVISIÓN
}

\author{
REMOVAL OF LEAD AND NICKEL IN AQUEOUS \\ SOLUTIONS USING LIGNOCELLULOSIC BIOMASS: \\ A REVIEW
}

\begin{abstract}
Edgar Quiñones ${ }^{1}$, Candelaria Tejada ${ }^{2}$, Cesar Arcia ${ }^{3}$, Víctor Ruiz ${ }^{4}$
${ }^{1}$ Ing.Civil. Ph.D. en Ingeniería Ambiental. Docente de la Facultad de Ingeniería, Universidad de Cartagena, 130006. Avenida el Consulado calle 30 No 48-152, Cartagena-Bolívar, Colombia, garedbolanos@hotmail.com; ${ }^{2}$ Ing. Química. Magister en Educación. Candidata a Magíster en Ingeniería Ambiental. Docente Universidad de Cartagena, calle 30 \# 50-100, CartagenaBolívar, Colombia, ctejadat@unicartagena.edu.co; ${ }^{3}$ Ing. Civil. Especialista en Ingeniería Sanitaria y Ambiental, Universidad de Cartagena, 130006, Cartagena-Bolívar, Colombia, arcialafont@yahoo.com; ${ }^{4}$ Ing. Civil. Especialista en Ingeniería Sanitaria y Ambiental, Universidad de Cartagena, 130006, Cartagena-Bolívar, Colombia, v_ruiz_r@yahoo.com
\end{abstract}

Rev. U.D.C.A Act. \& Div. Cient. 16(2): 479-489, Julio-Diciembre, 2013

\section{RESUMEN}

En la presente revisión, se identifican los bioadsorbentes extraídos de biomasas residuales utilizadas en la remoción de dos metales pesados, que presenta una amplia toxicidad para el ambiente: plomo y níquel. Se evalúa la capacidad de remoción de los mismos, destacando aquellos en los que se han obtenido altos porcentajes de remoción, mostrando la cinética aplicada en estos experimentos. Se encontró, que los bioadsorbentes más usados incluyen residuos de madera, cáscaras de frutos secos, residuos de cereales y cítricos. Para la remoción de plomo (II), el bagazo de caña de azúcar, con una capacidad de remoción de $333 \mathrm{mg} / \mathrm{g}$ y para el níquel (II), la corteza de Acacia, con una capacidad de remoción de 294,1mg/g, han sido los bioadsorbentes con mayor eficiencia de remoción. Se encuentra que, en la mayoría de los experimentos, la cinética del proceso de adsorción es regida por la ecuación cinética de pseudo-segundo orden. Se recomienda el diseño de plantas piloto para la remoción de iones metálicos con las biomasas que mostraron mayor capacidad de adsorción, con el fin de que estos procesos puedan ser llevados a escala industrial.

Palabras clave: Adsorción, bioadsorbente, ion metálico.

\section{SUMMARY}

The present review identifies bioadsorbents, extracted from residual biomass used in the removal of two highly toxic heavy metals in the environment: lead and nickel. The ability of removal was evaluated, highlighting those which showed removal, presenting the kinetics applied in these experiments. The bioadsorbente used include wood waste, nut shells, grain waste and citrus. It was found that for the removal of lead(II), the sugar cane bagasse with a removal capacity of $333 \mathrm{mg} / \mathrm{g}$ and for nickel (II) acacia bark with a removal capacity of $294.1 \mathrm{mg} / \mathrm{g}$, stand out. These bioadsorbents are those reported with efficient removal ability. The kinetics of the adsorption process in most of the revised experiments is governed by the rate equation of pseudo-second order. A design of a pilot plant for removal of metal ions with biomasses with high adsorption capacity is recommended in order that these processes can be carried out in an industrial scale.

Key words: Adsorption, bioadsorbent, metal ion.

\section{INTRODUCCIÓN}

Como constituyente importante de muchas aguas residuales industriales, se encuentran metales pesados, aunque su cuantificación sea a niveles de trazas (Ahluwalia \& Goyal, 2007). Cualquier catión que tenga un peso atómico superior a 23, se considera un metal pesado; entre ellos, se puede destacar el níquel, manganeso, plomo, cromo, cadmio, zinc, cobre, hierro, mercurio, entre otros (Alonso, 2008; Cavaco et al. 2007). Todos ellos, sustancias contaminantes que se deben tener obligatoriamente en consideración, para fijar valores límites de emisiones, aunque algunos en pequeñas cantidades son imprescindibles para el normal desarrollo de 
la vida biológica, por ejemplo, el crecimiento de las algas (Manzoor et al. 2006; Mohan \& Pittman, 2006).

El incremento en el uso de sustancias químicas en los procesos industriales ha resultado en la generación de grandes cantidades de efluentes acuíferos, que contienen altos niveles de contaminantes orgánicos e inorgánicos, lo cual, causa severos daños a la vida acuática, además, las sales de estos son solubles enagua y, por tanto, no pueden ser separadas por métodos ordinarios (Hussein et al. 2004); precisamente, por tal razón surge la bioadsorción como alternativa (PinzónBedoya \& Vera-Villamizar, 2009).

La adsorción es un proceso mediante, el cual, se extrae materia de una fase y se concentra sobre la superficie de otra. La sustancia que se concentra en la superficie se llama "adsorbato" y la fase sobre la que se da el proceso "adsorbente"; en este proceso debe existir afinidad del adsorbente por los adsorbatos, con el fin de que estos últimos sean transportados hacia el sólido, donde van a ser retenidos. Si las moléculas penetran al interior de la fase sólida, el proceso se conoce como absorción, mientras que el término sorción, generalmente es utilizado cuando los procesos de adsorción y absorción ocurren simultáneamente y no se pueden distinguir uno de otro (Dabrowski, 2001; Cañizares-Villanueva, 2000).

En cuanto a la cinética de la adsorción, describe la velocidad de atrapamiento del adsorbato, lo que controla el tiempo de residencia en la interfase sólido-disolución del efluente tratado (Ho \& Mckay, 1999; Cheung et al. 2000). Para describir la cinética del proceso de adsorción, se deben tener en cuenta las siguientes etapas: la transferencia de masa del ión metálico desde el seno de la solución hacia la superficie del adsorbente; la adsorción del ion metálico y la difusión interna del ión metálico en el adsorbente. Para ello, se usan los modelos que se detallan a continuación: el primero es el modelo de Primer Orden Reversible, que se basa en la suposición que a cada ión metálico se le asigna un sitio de adsorción del material adsorbente y, por tanto, el mecanismo y la velocidad de adsorción dependerá de varios factores como: la presencia de otro ion en la solución, la selectividad, tipo y, en general, características del adsorbente. En términos de velocidad de reacción, el modelo se expresa como:

$$
\frac{d_{t}}{d t}=k_{7} *\left(q_{1}-q_{t}\right)
$$

Donde $q_{t}(\mathrm{mmol} / \mathrm{g})$ es la cantidad adsorbida en un tiempo $t$, $\mathrm{q}_{\mathrm{e}}(\mathrm{mmol} / \mathrm{g})$ es la cantidad adsorbida en el equilibrio y $\mathrm{k}_{1}$ (1/ min) es la constante cinética de primer orden.

El Modelo de Pseudo Segundo Orden, desarrollado por Ho \& McKay (1999), supone que el adsorbato se adsorbe en dos sitios activos de la biomasa, por tanto, el mecanismo controlante del fenómeno es la velocidad de adsorción del ion metálico en el adsorbente y no la transferencia de masa. En términos de velocidad de reacción, se expresa como:

$$
\frac{d_{q_{4}}}{d}=k_{2} *\left(q_{4}-q_{t}\right)^{2}
$$

Integrando la ecuación anterior y manteniendo constantes las condiciones de contorno descritas para este modelo, se tiene:

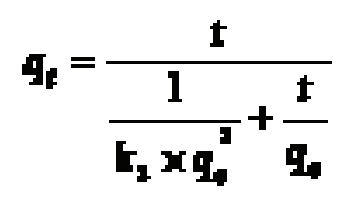

Donde $\mathrm{k}_{2}$ es la constante cinética de Pseudo Segundo Orden ( $\mathrm{g} / \mathrm{mmol}$.min) y $\mathrm{h}=\mathrm{k}_{2}{ }^{*} \mathrm{q}_{\mathrm{e}}{ }^{2}$ siendo $\mathrm{h}$ ( $\left.\mathrm{mmol} / \mathrm{g} . \mathrm{min}\right)$ la velocidad inicial de adsorción.

Otro de los modelos usados es el de Elovich, el cual, supone que los sitios activos del bioadsorbente son heterogéneos y, por ello, exhiben diferentes energías de activación, basándose en un mecanismo de reacción de segundo orden, para un proceso de reacción heterogénea. En términos de velocidad de reacción, se expresa como:

$$
n_{t}=\frac{1}{f} \ln (a p)+\frac{1}{\beta} \ln
$$

Donde $\alpha$ (mmol/gmin) es la velocidad inicial de adsorción y $\beta$ está relacionado con el grado de cobertura de la superficie y la energía de activación implicada en la quimiosorción (g/ mmol) (Cheung et al. 2000).

Por último, también se tiene en cuenta el modelo de Difusión Intraparticular, que establece un proceso controlado por la adsorción en los poros, en el que: la velocidad inicial es directamente proporcional a la concentración del soluto, se caracteriza por la dependencia entre la adsorción específica y la raíz cuadrada del tiempo, siendo la pendiente la velocidad de difusión (Weber \& Morris, 1963). Con base en lo anterior, la ecuación que define la difusión intraparticular viene dada por:

$$
g_{\mathrm{r}}=\mathrm{k} * \sqrt{\mathrm{t}}
$$

Donde $\mathrm{k}\left(\mathrm{mmol} \cdot \mathrm{g}^{-1} \cdot \mathrm{min}^{-1 / 2}\right)$ es la constante de velocidad de difusión intraparticular.

La actividad industrial y minera supone una preocupación importante en salud pública, por su toxicidad aguda y por la amplia variedad de fuentes de exposición, al arrojar al ambiente metales tóxicos, como el plomo y el níquel. Esta situación ha conducido a la búsqueda de tecnologías para 
resolver este problema, utilizando métodos tradicionales que, en su mayoría, son de alto costo e ineficiencia; sin embargo, surgen alternativas, como la adsorción con biomasa, que es de bajo costo y amigable con el ambiente, puesto que ofrece la posibilidad de recuperar desechos agrícolas e industriales para su posterior uso, generándose un valor agregado, que contribuye a la sustentabilidad del sistema. Es por las razones mencionadas, que en el presente trabajo se realiza una revisión de la literatura articulada con el uso de diferentes biomasas vegetales residuales para remover metales pesados, efectuando una descripción de las más utilizadas en la remoción de plomo y de níquel en aguas residuales, identificando aquellas con las que se han obtenido mejores resultados.

\section{MATERIALES Y MÉTODOS}

La búsqueda de artículos, se realizó en las bases de datos de ScienceDirect, con palabras clave, tales como biosorption, adsorption, remotion, heavy metal y los símbolos atómicos de los metales ( $\mathrm{Pb}, \mathrm{Ni})$. Los resultados fueron clasificados, teniendo en cuenta que fueran de los últimos seis años y que en el artículo publicado, se estudiara el uso de biomasa residual vegetal. Se organizaron las biomasas encontradas, destacando en una tabla aquellas con las que se obtuvieron mejores resultados, identificando el tipo de biomasa, el metal que remueve, la capacidad de adsorción y la referencia bibliográfica, con el fin de estudiar aquellas, con las cuales, se pueda escalar un proceso de adsorción de metales pesados, en Colombia.

Además, se identificaron las ecuaciones cinéticas usadas en los artículos consultados, que permiten modelar la cinética de adsorción de cinco metales: plomo, níquel, cadmio, cromo y mercurio, para determinar cuál de las ecuaciones es la más aplicable y, en general, la que mejor describe el proceso de adsorción con biomasa residual.

\section{RESULTADOS Y DISCUSIÓN}

Biomasas usadas en la remoción de plomo: Al revisar los artículos consultados, se encuentra que son diversas las biomasas lignocelulósicas residuales usadas para la remoción de metales pesados, en aguas contaminadas.

Bulut \& Tez (2007), utilizando aserrín de madera de Nogal (Juglans regia), mostraron que la máxima adsorción de $\mathrm{Pb}$ (II) en solución se obtuvo con un tiempo de contacto de $60 \mathrm{~min}$, una concentración inicial del metal de 200mg/L, una temperatura de $25^{\circ} \mathrm{C}$ y una velocidad de agitación de 150rpm. Además, la ecuación de la cinética que mejor ajustó los datos experimentales fue la de pseudo-segundo orden con un $\mathrm{R}^{2}$ de 0,9996 ; los autores reportaron una máxima adsorción de $\mathrm{Pb}$ (II) de 3,78mg/g.

El potencial de biosorción de la corteza de pino (Pinus bru- tia) para la eliminación de iones $\mathrm{Pb}$ (II) fue evaluada por Gundogdua et al. (2009), quienes obtuvieron la máxima capacidad de adsorción de 76,8mg/g, con un $\mathrm{pH}$ de 4 , un tiempo de 4 horas, una concentración inicial del metal entre 50 y $1000 \mathrm{mg} / \mathrm{L}$ y una masa de la corteza de $1 \mathrm{~g} / \mathrm{L}$; los autores reportaron que el modelo cinético que mejor ajustó los datos experimentales fue el de pseudo-segundo orden, con un coeficiente de correlación $\mathrm{R}^{2}=0,999$. Por su parte, Taty-Costodes et al. (2003) también estudiaron el pino (Pinus sylvestris), usando aserrín, encontrando una capacidad de adsorción de 9,8mg/g, con un pH de 5,5 y 30 minutos de tiempo de contacto. El proceso siguió la cinética de pseudosegundo orden, con un coeficiente de correlación $\mathrm{R}^{2}$ de 0,91.

Rafatullaha et al. (2009) trabajaron el aserrín de meranti (Acuminata shorea) para la eliminación de $\mathrm{Pb}$ (II) en solución; la máxima adsorción se obtuvo a pH 6, con un tiempo de 120 min, una dosis de adsorbente de $10 \mathrm{~g} / \mathrm{L}$, una temperatura de $30^{\circ} \mathrm{C}$ y una velocidad de agitación de $100 \mathrm{rpm}$. La cinética de adsorción que se ajustó fue la de pseudo-segundo orden, con un $\mathrm{R}^{2}$ de 0,999 , obteniéndose una máxima adsorción de $34,246 \mathrm{mg} / \mathrm{g}$.

La adsorción de $\mathrm{Pb}$ (II) con residuos de biomasa de algodonero (Gossypium hirsutum) fue estudiada por Riaza et al. (2009); ellos indican que una dosis de 0,20g de biomasa, pH 5 y 100mg/L de concentración inicial de $\mathrm{Pb}$ (II) son las condiciones óptimas para obtener la mayor bioadsorción, la cual, fue de 45,01 mg/g. El modelo que mejor ajustó los datos experimentales fue el de pseudo-segundo orden, con un $\mathrm{R}^{2}=0,997$.

De lo anterior, se puede resaltar que el modelo cinético que mejor ajusta los datos obtenidos de biomasa residual lignocelulósica, como el aserrín o similares, es el de pseudo-segundo orden y la que mejor remueve los iones de $\mathrm{Pb}$ (II) es la corteza de pino (Pinus brutia).

Usando cáscara de maní (Arachis hypogaea) para remover $\mathrm{Pb}$ (II), Qaiser et al. (2009) encontraron una máxima capacidad de biosorción de $31,54 \mathrm{mg} / \mathrm{g}$, con un $\mathrm{pH}$ óptimo para la eliminación de plomo de 5. También Shih-Wei et al. (2011) describieron la adsorción, mediante el empleo de ceniza de cáscara de maní; se encontró una remoción máxima de $33 \mathrm{mg} / \mathrm{g}$, bajo las siguientes condiciones: $3 \mathrm{~g} / \mathrm{L}$ de adsorbente, $100 \mathrm{mg} / \mathrm{L}$ de plomo inicial, $\mathrm{pH} 4,2$ y $30^{\circ} \mathrm{C}$ de temperatura. Se puede afirmar, que la ceniza de la cáscara de maní tiene mejor desempeño para remover $\mathrm{Pb}(\mathrm{II})$, que la cáscara sin ninguna modificación.

Karnitz \& Alves (2009) reportaron la preparación de nuevos materiales quelantes derivados del bagazo de caña de azúcar, para la adsorción de $\mathrm{Pb}$ (II). El material bioadsorbente, se preparó mediante dos modificaciones: la primera, fue el 
bagazo de caña de azúcar mercerizado con $\mathrm{NaOH} 5 \mathrm{~mol} / \mathrm{L}$ y, el otro material, se obtuvo de hacerlo reaccionar con dianhídrido etilendiaminotetraacético (EDTAD). Estos mostraron capacidades de adsorción de $\mathrm{Pb}(\mathrm{II})$, que van desde 192 a $333 \mathrm{mg} / \mathrm{g}$. Los materiales modificados con la mercerización mostraron las máximas capacidades comparados con los no mercerizados, siendo con el pH de 5,3 donde se obtuvo el mayor porcentaje de remoción.

El potencial de la cáscara de manzana blanca (Chrysophyllum albidum) para la remoción de $\mathrm{Pb}(\mathrm{II})$, resultó en que el proceso es altamente dependiente del $\mathrm{pH}$, siendo el de 7 un pH óptimo, con el cual, se obtuvo una remoción máxima de 8,5mg/g. La ecuación cinética de adsorción no fue referenciada en este estudio (Onwu \& Ogah, 2010).

García-Rosales \& Colín-Cruz (2010) reportan la eliminación de $\mathrm{Pb}$ (II) con tallos de maíz (Zea mays) y demostraron que el modelo cinético de pseudo-segundo orden fue el que mejor representó el proceso, con una capacidad de adsorción máxima de $80 \mathrm{mg} / \mathrm{g}$ y un $\mathrm{pH}$ óptimo de 6 .

Chen et al. (2010), para la eliminación del Pb(II) de soluciones acuosas estudiaron las hojas de alcanforero (Cinnamomum camphora). La capacidad máxima de adsorción de este biomaterial fue de $73,15 \mathrm{mg} / \mathrm{g}$, con un $\mathrm{pH}$ de 5 y un tiempo de contacto de 60min. Para este fin, Chakravarty et al. (2010) propusieron el uso de las hojas de bael (Aegle marmelos), lográndose una máxima adsorción de $104 \mathrm{mg} / \mathrm{g}$, a una concentración de $\mathrm{Pb}$ (II), de 50mg/L, un pH de 5,1, una dosis óptima de hojas de bael de $0,2 \mathrm{~g}$ y una temperatura de $25^{\circ} \mathrm{C}$. La ecuación cinética que mejor ajustó los datos experimentales en este estudio fue de pseudo-segundo orden, con un $\mathrm{R}^{2}=0,99$. Todavía, otra investigación fue realizada por Anwar et al. (2010), quienes investigaron la adsorción de $\mathrm{Pb}$ (II) sobre las cáscaras de plátano (Musa paradisiaca); los parámetros considerados fueron la dosis de adsorbente, $\mathrm{pH}$ de la solución, tiempo y velocidad de agitación. La adsorción máxima, según la isoterma de Langmuir, fue de $2,18 \mathrm{mg} / \mathrm{g}$.

Lasheen et al. (2012), al estudiar el uso de cáscara de naranja (Citrus sinensis), modificada con ácido nítrico como adsorbente en la remoción de $\mathrm{Pb}(\mathrm{II})$, encontrando que la cinética de la adsorción sigue el modelo de Pseudo-segundo orden. Se halló que la máxima remoción de $\mathrm{Pb}$ (II) fue de 73,5mg/g. También, Tapia et al. (2003) muestran la cáscara de naranja como un bioadsorbente con gran potencial, esta vez, modificadas con $\mathrm{NaOH}$ 0,2M; además, para reforzar la resistencia mecánica, los autores entrecruzaron la cáscara con $\mathrm{CaCl}_{2}$ 0,2M, hallando una máxima adsorción de 141,1mg/g, con un pH óptimo de 5. La velocidad del proceso de biosorción fue entre 10 y 24 horas. Se observa, con el tratamiento de desmetoxilación de la pectina, que si se encuentran en gran cantidad en la naranja, se aumenta la remoción del metal.
Se concluye que el énfasis de las investigaciones en la remoción de plomo se ha encaminado hacía la búsqueda de materiales agrícolas residuales, permitiendo el uso de biomasas abundantes, en países del trópico, como el bagazo de caña de azúcar, los tallos de maíz y la cáscara de naranja, lo que puede llevar a la bioadsorción a ser una tecnología económica, que contribuya al control eficaz y a la prevención de la contaminación por metales pesados del recurso hídrico. Se recomienda la búsqueda de nuevos materiales de desecho y el estudio de condiciones necesarias, para elevar los porcentajes de remoción de los mismos, teniendo en cuenta las modificaciones físicas y químicas de estos materiales, ya que se ha reportado que mejoran su capacidad de adsorción.

Se encontró, que el mecanismo de adsorción se ve afectado por la concentración inicial, ya que a medida que la concentración aumenta el porcentaje de adsorción disminuye. También cumplen un papel fundamental el tamaño de partícula y la agitación, puesto que a menor tamaño de partículas y mayor velocidad de agitación, se produce un aumento en el porcentaje de adsorción. Además, se concluye que el pH óptimo de adsorción de plomo, se encuentra entre 5 y 6 , lo que también influye en la especiación del ión metálico en solución.

Revisión de biomasas usadas en la remoción de níquel: Un alto grado de exposición a los compuestos solubles de níquel puede provocar graves problemas a la salud y aun cuando existen estrictas regulaciones sobre vertimientos; las industrias, en su mayoría metalmecánicas, descargan en sus efluentes grandes cantidades de níquel, por tal razón, el creciente interés por la producción de nuevos materiales capaces de remover este metal, que además sean de bajo costo, tales como la biomasa renovable.

Muhammad et al. (2007) investigaron la adsorción de Ni(II) con salvado de arroz (Oryza sativa), protonado con tres ácidos: $\mathrm{HCl}, \mathrm{H}_{2} \mathrm{SO}_{4}, y \mathrm{H}_{3} \mathrm{PO}_{4}$, obteniéndose una adsorción de $102 \mathrm{mg} / \mathrm{g}$, con un $\mathrm{pH}$ de 6 ; la ecuación cinética que mejor describió los datos experimentales fue la de pseudo-segundo orden, con un coeficiente de correlación $\mathrm{R}^{2}=1$. Otros autores experimentaron la biosorción de iones de Ni(II) con corteza de acacia (Acacia leucocephala), logrando el mejor ajuste de los datos con la ecuación de pseudo-segundo orden; la máxima capacidad fue de $294,1 \mathrm{mg} / \mathrm{g}$ a $30^{\circ} \mathrm{C}$, con un tiempo de contacto de 120min y pH 5 (Venkata et al. 2009).

Nuhoglu \& Malkoc (2009) evaluaron la adsorción de Ni(II) usando residuos de aceite de oliva, encontrando que la máxima adsorción fue de $14,8 \mathrm{mg} / \mathrm{g}$, a $60^{\circ} \mathrm{C}$ y $\mathrm{pH}$ de 4 ; además, los datos siguieron el modelo cinético de pseudo-segundo orden.

El uso del aserrín de meranti (Acuminata shorea) en la eliminación de iones de Ni(II) mostró una adsorción de 200mg/L; 
los autores lograron la máxima adsorción a pH 6, con un tiempo de contacto de 120min, una dosis de adsorbente de $10 \mathrm{~g} / \mathrm{L}$, una temperatura de $30^{\circ} \mathrm{C}$ y una velocidad de agitación de 100rpm. La ecuación de la cinética de mejor ajuste fue la de pseudo-segundo orden, obteniéndose la máxima adsorción de $\mathrm{Ni}(\mathrm{II})$ de 35,97mg/g (Rafatullaha et al. 2009).

Srivastava et al. (2009) emplearon la cáscara de arroz como bioadsorbente en la remoción de $\mathrm{Ni}(\mathrm{II})$ y hallaron que la ceniza de esta fue efectiva, con un mayor porcentaje de eliminación de iones metálicos cuando la concentración inicial de adsorbato en la solución era baja. Se obtuvo una remoción máxima de $0,083 \mathrm{mg} / \mathrm{g}$ del ion metálico.

Zaheer et al. (2010) estudiaron la capacidad de adsorción de las hojas del árbol de Buda (Ficus religiosa) tratadas con ácido. Los resultados mostraron que el equilibrio de adsorción se estableció en 60 minutos, con una capacidad de $6,35 \mathrm{mg} / \mathrm{g}$ y dependió, en gran medida, del pH. También, se observó que la adsorción de Ni(II) disminuía con el aumento de la temperatura. La cinética de adsorción fue descrita por el modelo de ecuación cinética de pseudo-segundo orden. Otros autores investigaron la biosorción de Ni(II) con paja de cebada, que mostró una máxima adsorción de 35,81 mg/g, con un pH de 4,85. En este trabajo, la ecuación de velocidad cinética no fue presentada (Thevannan et al. 2010).

La adsorción de Ni(II) utilizando cáscara de granada (Punica granatum) fue descrita por Bhatnagara \& Minochaa (2010); la máxima capacidad de sorción fue de $52 \mathrm{mg} / \mathrm{g}$, en un tiempo de contacto de 7 horas, a una temperatura de $45^{\circ} \mathrm{C}$. La ecuación de pseudo-segundo orden modeló bien el proceso.

Anoop et al. (2011) analizaron la adsorción de Ni(II) empleando carbón activado a partir de bagazo de caña. Las condiciones óptimas para la extracción máxima del metal, se dieron a un $\mathrm{pH}$ de 6,5, con el que se obtuvo una remoción máxima de 140,85mg/g. Acevedo et al. (2007), también usaron el bagazo de caña (Saccharum officinarum), modificándola con $\mathrm{H}_{3} \mathrm{PO}_{4} 40 \% \mathrm{p} / \mathrm{p}$, durante 14 horas, el material fue pirolizado por una hora, a 400 y $500^{\circ} \mathrm{C}$, obteniendo dos tipos de carbón para la remoción del metal, en una mina carbonífera. La máxima remoción de 85,66\%, se logró con el carbón activado a $500^{\circ} \mathrm{C}$, el pH óptimo fue de 5,5. La máxima remoción en el efluente industrial fue de $96,03 \%$.

Igualmente, Aloma et al. (2012) evaluaron el bagazo de caña de azúcar, obteniéndose la máxima capacidad de sorción de $2 \mathrm{mg} / \mathrm{g}$ de $\mathrm{Ni}(\mathrm{II})$, a $25^{\circ} \mathrm{C}$, con un $\mathrm{pH}$ de 5 . Estos autores encontraron que el proceso de adsorción se ajustó al modelo de pseudo-segundo orden. La activación de este material mejoró, en gran medida, su efectividad de adsorción.

El uso de la cáscara de naranja sin modificar y modificada por copolimerización injertada, para la eliminación de iones de $\mathrm{Ni}(\mathrm{II})$ fue evaluada por Feng et al. (2011). La capacidad máxima de adsorción de este biomaterial fue de $162,6 \mathrm{mg} / \mathrm{g}$, para la cáscara modificada y de 9,82, sin modificar, a $\mathrm{pH}$ 5,5, con un tiempo de contacto de 150 min y una dosis de adsorbente de $50 \mathrm{mg} / \mathrm{L}$. La ecuación de pseudo-segundo orden fue la que mejor ajustó los datos, con un $\mathrm{R}^{2}$ de 0,9936.

Harikishore et al. (2011) estudiaron la corteza de Moringa (Moringa oleífera), un residuo agrícola para la obtención de un sorbente en la eliminación de Ni(II). La capacidad máxima de bioadsorción fue de $30,38 \mathrm{mg} / \mathrm{g}$, con un $\mathrm{pH}$ óptimo de la solución de 6 , una concentración de biomasa de $0,4 \mathrm{~g}$ y un tiempo de contacto de 60min. Los estudios cinéticos mostraron que el modelo de pseudo-segundo orden describe bien los datos experimentales de adsorción. Los mismos autores (2012) estudiaron la bioadsorción de Ni(II) con polvo de hojas de la moringa, modificadas químicamente con $\mathrm{NaOH}$ y ácido cítrico. La capacidad máxima que se obtuvo fue de $163,88 \mathrm{mg} / \mathrm{g}$, con un $\mathrm{pH}$ de 6 , a una temperatura de $49,84^{\circ} \mathrm{C}$, en un tiempo de contacto de $50 \mathrm{~min}$.

Al estudiar los parámetros de adsorción de níquel, al igual que en el caso del plomo, la concentración inicial del metal, el tamaño de partículas y el pH, juegan un papel predominante en el proceso de adsorción, tal que, a medida que incrementa la concentración inicial del ion y el tamaño de partícula, se disminuye el porcentaje de adsorción del metal.

De la revisión realizada, se puede observar que en los últimos seis años se ha investigado el uso de una amplia variedad de biomasas para la remoción de los iones metálicos plomo y níquel en aguas contaminadas, entre los que se incluye cortezas, residuos de madera, hojas de diferentes arboles, cáscaras de frutos secos, residuos de cereales y cítricos. En la tabla 1 , se muestra un resumen de las biomasas más eficientes para la remoción de ambos metales.

Se tiene que para la remoción de $\mathrm{Pb}(\mathrm{II})$, el bagazo de caña de azúcar (S. officinarum), con una capacidad de remoción de $333 \mathrm{mg} / \mathrm{g}$ (Karnitz \& Alves, 2009) y para el níquel (II), la corteza de acacia (A. leucocephala), con una capacidad de remoción de 294,1mg/g (Venkata et al. 2009), presentan la máxima capacidad de adsorción reportada.

Se destaca que el bagazo de caña de azúcar fue tratado con hidróxido de sodio, tratamiento que produce cambios en la estructura de las fibras que mejoran las propiedades de accesibilidad de la biomasa y, de hecho, reaccionar con dianhídrido etilendiaminotetraacético; en general, ambos procesos modifican los grupos químicos presentes en la caña, aumentando su capacidad de adsorción, convirtiéndose en materiales más eficientes y con mayor potencial, para un posible uso a escala industrial. 
Tabla1. Capacidad de remoción de las biomasas.

\begin{tabular}{|c|c|c|c|}
\hline Biomasa utilizada & Metal removido & $\begin{array}{c}\text { Capacidad } \\
\text { remoción } \mathbf{( m g / g )}\end{array}$ & Ref. Bibliográfica \\
\hline Hojas de Moringa (M. oleifera) & $\mathrm{Ni}$ (II) & 163,88 & Harikishore et al. 2012 \\
\hline Cáscara de Naranja (C. sinensis) & $\mathrm{Ni}$ (II) & 162,6 & Feng et al. 2011 \\
\hline Corteza de Acacia (A. leucocephala) & $\mathrm{Ni}$ (II) & 294,1 & Venkata et al. 2009 \\
\hline Hojas de Bael (A. marmelos) & $\mathrm{Pb}(\mathrm{II})$ & 104 & Chakravarty et al. 2010 \\
\hline Bagazo de Caña de Azúcar & $\mathrm{Pb}(\mathrm{II})$ & 333 & Karnitz \& Alves, 2009 \\
\hline Tallos de Maíz (Z. mays) & $\mathrm{Pb}(\mathrm{II})$ & 80 & García \& Colín, 2010 \\
\hline
\end{tabular}

De lo anterior, se observa que ninguna biomasa muestra resultados excelentes para ambos tipos de metal; ejemplo de esta situación, es el bagazo de caña, puesto que para el plomo ha sido la biomasa que mayor eficiencia de remoción presenta, mientras que en el níquel, no se alcanza ni la mitad del porcentaje.

La biosorción se convierte en una técnica promisoria para la remoción de metales pesados de ambientes acuosos, especialmente, con adsorbentes derivados de materiales lignocelulósicos, que cuentan con una amplia variedad de polímeros en su estructura, como: celulosa, hemicelulosa, pectina, lignina y proteínas, que los hacen biomateriales eficientes, siendo una tecnología limpia y barata para el tratamiento de efluentes contaminados a concentraciones tan bajas, como 1mg/L (Osman et al. 2010).

Comparación de la cinética de los procesos de adsorción: Se decidió indagar por el tipo de cinética usada para varios tipos de metales pesados $(\mathrm{Cr}, \mathrm{Pb}, \mathrm{Ni}, \mathrm{Cd}$ y Hg), con el fin de determinar la ecuación que mejor ajusta los datos experimentales trabajados, con diferentes biomasas. En la tabla 2, se observa un resumen de la información encontrada. Del estudio de la cinética usada por los procesos revisados, no sólo con el plomo y el níquel, sino también con mercurio, cromo y cadmio, es la ecuación de pseudo-segundo orden, reportando un mejor ajuste de los datos experimentales, con coeficientes de correlación superiores a la de otros modelos ensayados. Por lo tanto, el mecanismo controlante de la velocidad de adsorción no depende de la transferencia de masa, sino de la reacción de adsorción del ion metálico.

Tendencia de investigación en relación con la remoción de plomo y níquel: Al revisar la tendencia, en cuanto a la investigación de las biomasas, se identifican tres de las más trabajadas contra el número de artículos reportados en los últimos seis años; estos datos fueron tomados de la base de datos Sciverse-Scopus. Las gráficas 1 y 2 reportan los datos encontrados para la remoción de níquel y plomo, respectivamente, para los residuos de naranja, trigo y arroz.

De las gráficas 1y 2 , se deduce que son más de 2.000 artículos que tratan del uso de estos bioadsorbentes en la remoción de níquel y plomo, siendo la cáscara de arroz, el más estudiado; conjuntamente, se destaca que de las biomasas reportadas, para ambos metales, es el trigo la de la de menor contribución, siendo el níquel el metal menos trabajado comparado con el plomo; esta tendencia, se puede deber al ser el más tóxico y, por tanto, las concentraciones máximas permitidas son menores.

Se observa que son más de 700 artículos, en cuatro años, dedicados al estudio de los residuos de naranja para la remoción de plomo y de níquel, pudiendo aprovechar un desecho de la industria agrícola, abundante en países, como Colombia, para dar una solución adecuada al problema ambiental de la contaminación con efluentes que presentan altas concentraciones, de los metales en cuestión. De igual modo, se nota que aunque existen 500 artículos que evalúan, en cuatro años, lo referente a los residuos de trigo, estos son significativamente menores que los de cascara de naranja, quizás por la mayor efectividad de remoción reportada por los residuos de naranja.

Se puede concluir que, en los últimos seis años, se ha investigado el uso de una amplia variedad de biomasas lignocelulósicas residuales para la remoción de iones metálicos, en aguas contaminadas, entre los que se incluye cortezas de arboles, residuos de madera, semillas y hojas de diferentes árboles, cáscaras de frutos secos, residuos de cereales 
Tabla 2. Cinética del proceso de adsorción.

\begin{tabular}{|c|c|c|c|}
\hline Biomasa utilizada & Metal removido & Ecuación de cinética & Referencia Bibliográfica \\
\hline Aserrín & $\mathrm{Cr}(\mathrm{VI})$ & Pseudo $2^{\text {do }}$ Orden & Gupta \& Babu, 2009 \\
\hline Aserrín de Meranti & $\mathrm{Cr}$ (III) & Pseudo $2^{\text {do }}$ Orden & Rafatullaha et al. 2009 \\
\hline Aserrín de Meranti & $\mathrm{Pb}(\mathrm{II})$ & Pseudo $2^{\text {do }}$ Orden & Rafatullaha et al. 2009 \\
\hline Aserrín de Meranti & $\mathrm{Ni}(\mathrm{II})$ & Pseudo $2^{\text {do }}$ Orden & Rafatullaha et al. 2009 \\
\hline Aserrín de Nogal & $\mathrm{Pb}(\mathrm{II})$ & Pseudo $2^{\text {do }}$ Orden & Bulut \& Tez, 2007 \\
\hline Bagazo de Caña de Azúcar & $\mathrm{Ni}(\mathrm{II})$ & Primer Orden & Anoop et al. 2011 \\
\hline Bagazo de Caña de Azúcar & $\mathrm{Ni}(\mathrm{II})$ & Pseudo $2^{\text {do }}$ Orden & Aloma et al. 2012 \\
\hline Cáscara de Arroz & $\mathrm{Hg}$ (II) & Pseudo $2^{\text {do }}$ Orden & El-Shafey, 2010 \\
\hline Cáscara de Arroz & Cd (II) & Pseudo $2^{\text {do }}$ Orden & Ye et al. 2010 \\
\hline Cáscara de fríjol & Cd (II) & Pseudo $2^{\text {do }}$ Orden & Saeed et al. 2009 \\
\hline Cáscara de Granada & $\mathrm{Ni}(\mathrm{II})$ & Pseudo $2^{\text {do }}$ Orden & Bhatnagara \& Minochaa, 2010 \\
\hline Cáscara de Judía & $\mathrm{Hg}$ (II) & Pseudo $2^{\text {do }}$ Orden & Madhava et al. 2009 \\
\hline Cáscara de Maní & $\mathrm{Cr}(\mathrm{VI})$ & Pseudo $2^{\text {do }}$ Orden & Qaiser et al. 2009 \\
\hline Cáscara de Maní & $\mathrm{Cr}$ (III) & Pseudo $2^{\text {do }}$ Orden & Witek-Krowiak et al. 2011 \\
\hline Cáscara de Maní & $\mathrm{Pb}(\mathrm{II})$ & Pseudo $2^{\text {do }}$ Orden & Shih-Wei et al. 2011 \\
\hline Cáscara de Naranja & $\mathrm{Pb}(\mathrm{II})$ & Pseudo $2^{\text {do }}$ Orden & Lasheen et al. 2012 \\
\hline Cáscara de Naranja & $\mathrm{Ni}(\mathrm{II})$ & Pseudo $2^{\text {do }}$ Orden & Feng et al. 2011 \\
\hline Cáscara de Naranja & Cd (II) & Pseudo $2^{\text {do }}$ Orden & Lasheen et al. 2012 \\
\hline Cáscara de Nuez & $\mathrm{Hg}$ (II) & Pseudo $2^{\text {do }}$ Orden & Zabihi et al. 2010 \\
\hline Cáscara de Pistacho & $\mathrm{Cr}(\mathrm{VI})$ & Pseudo $2^{\text {do }}$ Orden & Moussavi \& Barikbin, 2010 \\
\hline Cáscara de Plátano & $\mathrm{Cr}(\mathrm{VI})$ & Primer Orden & Memon et al. 2009 \\
\hline Cáscara de Tamarindo & $\mathrm{Cr}(\mathrm{VI})$ & Primer Orden & Rao Popuri et al. 2007 \\
\hline Cáscara Fruta de Bael & $\mathrm{Cr}(\mathrm{VI})$ & Pseudo $2^{\text {do }}$ Orden & Anandkumar \& Mandal, 2009 \\
\hline Cáscara Semilla Ceiba & $\mathrm{Hg}$ (II) & Pseudo $2^{\text {do }}$ Orden & Madhava et al. 2009 \\
\hline Corteza de Acacia & $\mathrm{Ni}(\mathrm{II})$ & Pseudo $2^{\text {do }}$ Orden & Venkata et al. 2009 \\
\hline Corteza de Moringa & $\mathrm{Ni}(\mathrm{II})$ & Pseudo $2^{\text {do }}$ Orden & Harikishore et al. 2011 \\
\hline Corteza de Pino & $\mathrm{Pb}(\mathrm{II})$ & Pseudo $2^{\text {do }}$ Orden & Gundogdua et al. 2009 \\
\hline Fruta de Gular & $\mathrm{Cr}(\mathrm{VI})$ & Pseudo $2^{\text {do }}$ Orden & Rao \& Rehman, 2010 \\
\hline Hojas de Alcanforero & $\mathrm{Pb}(\mathrm{II})$ & Pseudo $2^{\text {do }}$ Orden & Chen et al. 2010 \\
\hline Hojas de Bael & $\mathrm{Pb}$ (II) & Pseudo $2^{\text {do }}$ Orden & Chakravarty et al. 2010 \\
\hline Hojas de Moringa & $\mathrm{Ni}(\mathrm{II})$ & Pseudo $2^{\text {do }}$ Orden & Harikishore et al. 2012 \\
\hline Hojas Ficus Religiosa & $\mathrm{Ni}(\mathrm{II})$ & Pseudo $2^{\text {do }}$ Orden & Zaheer et al. 2010 \\
\hline Residuos Aceite Oliva & $\mathrm{Ni}(\mathrm{II})$ & Pseudo $2^{\text {do }}$ Orden & Nuhoglu \& Malkoc, 2009 \\
\hline Salvado de Trigo & $\mathrm{Cr}(\mathrm{VI})$ & Pseudo $2^{\text {do }}$ Orden & Singh et al. 2009 \\
\hline Salvado de Arroz & $\mathrm{Ni}(\mathrm{II})$ & Pseudo $2^{\text {do }}$ Orden & Muhammad et al. 2007 \\
\hline Semilla de Algodón & $\mathrm{Pb}(\mathrm{II})$ & Pseudo $2^{\text {do }}$ Orden & Riaza et al. 2009 \\
\hline Tallos de Maíz & $\mathrm{Pb}$ (II) & Pseudo $2^{\text {do }}$ Orden & García \& Colín, 2010 \\
\hline
\end{tabular}




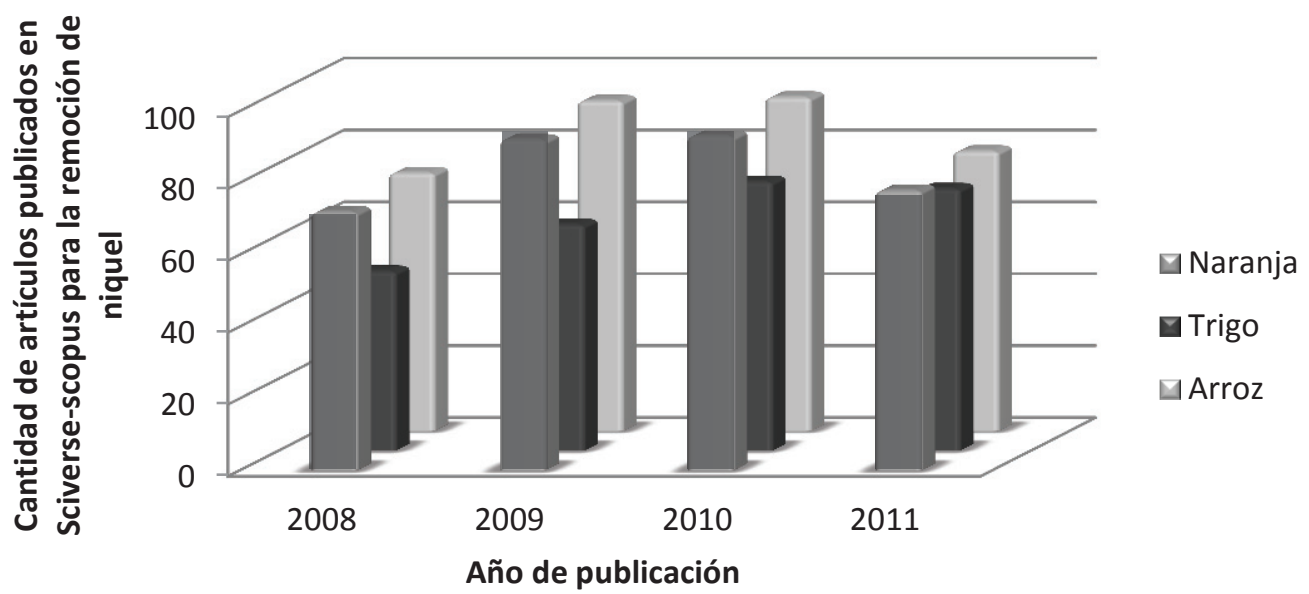

Gráfica 1. Artículos publicados sobre la adsorción de níquel con residuos de naranja, trigo y arroz.

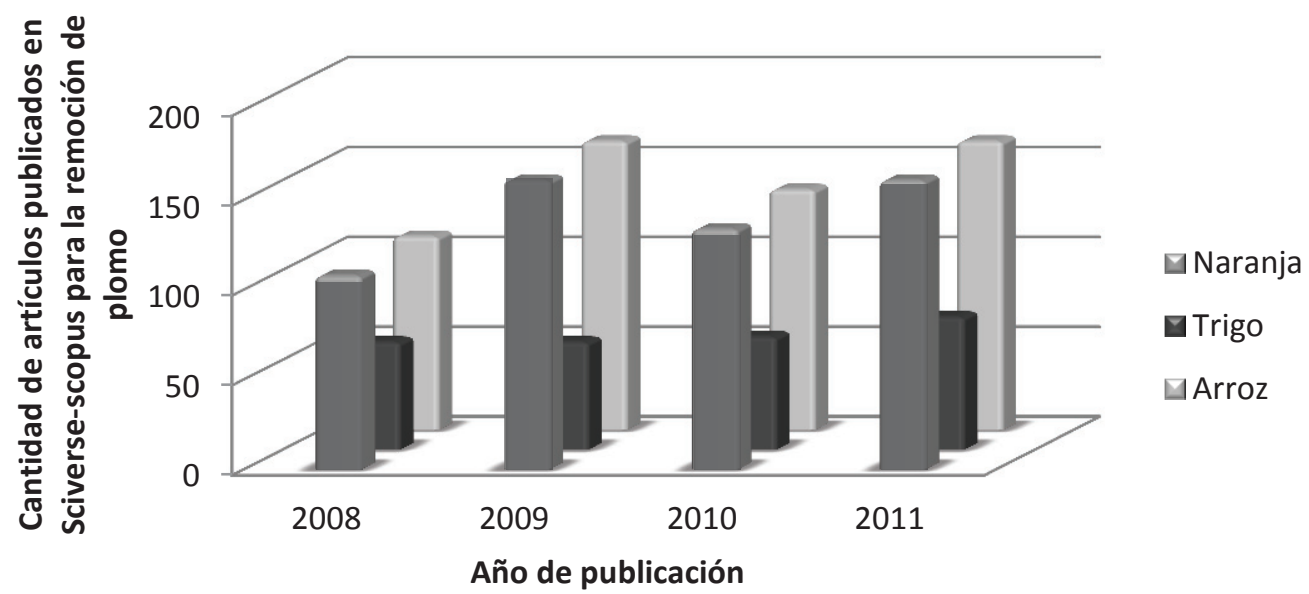

Gráfica 2. Artículos publicados sobre la adsorción de plomo con residuos de naranja, trigo y arroz.

y cítricos. Los residuos del arroz, de trigo y de naranja son los más empleados para remover níquel y plomo, siendo el bagazo de caña el de mayor capacidad de remoción, para el plomo y, la corteza de acacia, para el níquel. Cabe mencionar, que la tendencia observada fue pre-tratar químicamente la biomasa, para aumentar, en gran medida, su capacidad de remoción.

Se destaca, además, que la cinética del proceso de adsorción, en la gran mayoría de las biomasas estudiadas, es regida por la ecuación cinética de pseudo-segundo orden de Ho \& Mckay (1999), ya que con ella se obtuvieron coeficientes de correlación muy cercanos a 1, ajustándose mejor a los datos experimentales.

Se recomienda en base a las biomasas identificadas realizar trabajos de investigación de biomasas residuales, encontradas en regiones geográficas específicas; de igual forma, cuando se realicen experimentos con una biomasa determinada, se recomienda que el bioadsorbente obtenido, además de ser utilizado en su forma natural, sea también pre-tratado químicamente, para determinar en qué proporción aumenta su capacidad de remoción. Asimismo, diseñar plantas pilotos para remoción de iones metálicos con las bio- 
masas que mostraron mayor capacidad de remoción, a fin de desarrollar esta alternativa como una tecnología viable de implementar.

Conflictos de intereses: El manuscrito fue preparado y revisado con la participación de todos los autores, quienes declaramos que no existe ningún conflicto de intereses, que ponga en riesgo la validez de los resultados presentados.

\section{BIBLIOGRAFÍA}

1. ACEVEDO, S.; COLINA, M.; DÍAZ, A.; FERNÁNDEZ, N.; BEHLING, E.; MARÍN, J.; COLINA, G. 2007.Chemical activation of sugar cane bagasse (Saccharum officinarum) on nickel(II) adsorption in industrial effluents. Rev. Téc. Ing. Univ. Zulia. 30(3):244-252.

2. AHLUWALIA, S.S.; GOYAL, D. 2007. Microbial and plant derived biomass for removal of heavy metals from wastewater. Biores. Techn. 98:2243-2257.

3. ALOMA, L.; LARA, M.; RODRÍGUEZ, I.; BLAZQUEZ, G.; CALERO, M. 2012. Removal of nickel (II) ions from aqueous solutions by biosorption on sugarcane bagasse. J. Taiwan Inst. Chem. Eng. 43(2):275-281.

4. ALONSO, J.A. 2008. Los metales pesados en las aguas residuales. Disponible desde Internet en:http://www.madrimasd.org/blogs/remtavares/2008/02/02/83698 (con acceso 13/11/2012).

5. ANANDKUMAR, J.; MANDAL, B. 2009. Removal of Cr(VI) from aqueous solution using Bael fruit (Aegle marmelos Correa) shell as an adsorbent. J. Haz. Mat. 168:633-640.

6. ANOOP KRISHNAN, K.; SREEJALEKSHMI, K.G.; BAIJU, R.S. 2011. Nickel(II) adsorption onto biomass based activated carbon obtained from sugarcane bagasse pith. Biores. Techn. 102:10239-10247.

7. ANWAR, J.; SHAFIQUE, U.; ZAMAN, W.; SALMAN, M.; DAR, A.; ANWAR, S. 2010. Removal of $\mathrm{Pb}(\mathrm{II})$ and $\mathrm{Cd}$ (II) from water by adsorption on peels of banana. Biores. Techn. 101:1752-1755.

8. BHATNAGARA, A.; MINOCHAA, A. 2010. Biosorption optimization of nickel removal from water using $\mathrm{Pu}$ nica granatum peel waste. Colloids and Surfaces B: Biointerfaces. 76(2):544-548.

9. BULUT, Y.; TEZ, Z. 2007. Removal of heavy metals from aqueous solution by sawdust adsorption. J. Environ. Sci. 19:160-166.
10. CAÑIZARES-VILLANUEVA, R.O. 2000.Biosorción de metalespesados mediante el uso de biomasa microbiana. Rev. Latinoam. Microbiol. 42:131-143.

11. CAVACO, S.A.; FERNANDES, S.; QUINA, M.M.; FERREIRA, L.M. 2007. Removal of chromium from electroplating industry effluents by ion exchange resins. J. Haz. Mat. 144:634-638.

12. CHAKRAVARTY, S.; MOHANTY, A.; NAG SUDHA, T.; UPADHYAY, A.K.; KONAR, J.; SIRCAR, J.K.; MADHUKAR, A. 2010. Removal of $\mathrm{Pb}$ (II) ions from aqueous solution by adsorption using bael leaves (Aegle marmelos). J. Haz. Mat. 173:502-509.

13. CHEN, H.; ZHAO, J.; DAI, G.; WU, J.; YAN, H. 2010. Adsorption characteristics of $\mathrm{Pb}$ (II) from aqueous solution onto a natural biosorbent, fallen Cinnamomum camphora leaves. Desalination. 262:174-182.

14. CHEUNG, C.W.; PORTER, J.F.; McKAY, G. 2000. Elovich equation and modified second-order equation for sorption of cadmium ions onto bone char. J. Chem. Techn \& Biotechn. 75(11):963-970.

15. DABROWSKI, A. 2001. Adsorption-from theory to practice. Adv. Colloid and Interface Sci. 93:135-224.

16. EL-SHAFEY, E. 2010. Removal of Zn(II) and Hg(II) from aqueous solution on a carbonaceous sorbent chemically prepared from rice husk. J. Haz. Mat. 175:319327.

17. FENG, N.; GUO, X.; LIANG, S.; ZHU, Y.; LIU, J. 2011. Biosorption of heavy metals from aqueous solutions by chemically modified orange peel. J. Haz. Mat. 185(1):49-54.

18. GARCÍA-ROSALES, G.; COLÍN-CRUZ, A. 2010. Biosorption of lead by maize (Zea mays) stalk sponge. J. Environ. Manag. 91:2079-2086.

19. GUNDOGDUA, A.; OZDESA, D.; DURANA, C.; BULUT, V.; SOYLAKB, M.; SENTURKA, H. 2009. Biosorption of $\mathrm{Pb}$ (II) ions from aqueous solution by pine bark ( $\mathrm{Pi}$ nus brutia Ten). J. Chem. Eng. 153(1-3):62-69.

20. GUPTA, S.; BABU, B. 2009. Removal of toxic metal Cr(VI) from aqueous solutions using sawdust as adsorbent: Equilibrium, kinetics and regeneration studies. J. Chem. Eng. 150:352-365.

21. HARIKISHORE, D.; RAMANA, D.; SESHAIAH, K.; REDDY, 
A. 2011. Biosorption of $\mathrm{Ni}(\mathrm{II})$ from aqueous phase by Moringa oleifera bark, a low cost biosorbent. Desalination. 268(1-3):150-157.

22. HARIKISHORE, D.; REDDYA, K.; SESHAIAHA, K.; REDDYB, A.; LEE S. 2012. Optimization of Cd(II), $\mathrm{Cu}(\mathrm{II})$ and $\mathrm{Ni}(\mathrm{II})$ biosorption by chemically modified Moringa oleifera leaves powder. Carbohydrate Polymers. 88(3):1077-1086.

23. HO, Y.S.; MCKAY, G. 1999. Pseudosecond order model for sorption processes. Process Biochem. 34(5):451465.

24. HUSSEIN, H.; IBRAHIM, S.F; KANDEEL, K.; MOAWAD, H. 2004. Biosorption of heavy metals from waste water using Pseudomonas sp. Environ. Biotech. 7(1):1023-1027.

25. KARNITZ, O.; ALVES, L. 2009. Adsorption of Cu(II), $\mathrm{Cd}(\mathrm{II})$, and $\mathrm{Pb}$ (II) from aqueous single metal solutions by mercerized cellulose and mercerized sugarcane bagasse chemically modified with EDTA dianhydride (EDTAD). Carbohyd. Polym. 77:643-650.

26. LASHEEN, M.; AMMAR, N.; IBRAHIM, H. 2012. Adsorption/desorption of $\mathrm{Cd}(\mathrm{II}), \mathrm{Cu}(\mathrm{II})$ and $\mathrm{Pb}$ (II) using chemically modified orange peel: Equilibrium and kinetic studies. Solid State Sci. 14:202-210.

27. MADHAVA, M.; KUMAR, D.; VENKATESWARLU, P.; SESHAIAH, K. 2009. Removal of mercury from aqueous solutions using activated carbon prepared from agricultural by-product/waste. J. Environ. Manag. 90(1):634-643.

28. MANZOOR, S.; SHAH, M.; SHAHEEN, N.; KHALIQUE, A; JAFFAR, M. 2006. Multivariate analysis of trace metals in textile effluents in relation to groundwater. J. Haz. Mat. 137:31-37.

29. MEMON, J.; MEMON, S.; BHANGER, M.; EL-TURKI, A.; HALLAM, K.; ALLEN, G. 2009. Banana peel: A green and economical sorbent for the selective removal of $\mathrm{Cr}(\mathrm{VI})$ from industrial wastewater. Colloids and Surfaces B: Biointerfaces. 70:232-237.

30. MOHAN, D.; PITTMAN, C.U. 2006. Activated carbons and low cost adsorbents for remediation of triand hexavalent chromium from water. J. Haz. Mat. 137:762-811.

31. MOUSSAVI, G.; BARIKBIN, B. 2010. Biosorption of
chromium(VI) from industrial wastewater onto pistachio hull waste biomass. J. Chem. Eng. 162:893900.

32. MUHAMMAD, N.; RAZIYA, N.; MUHAMMAD, A. 2007. Biosorption of nickel from protonated rice bran. $\mathrm{J}$. Haz. Mat. 143(1-2):478-485.

33. NUHOGLU, Y.; MALKOC, E. 2009. Thermodynamic and kinetic studies for environmentaly friendly $\mathrm{Ni}(\mathrm{II})$ biosorption using waste pomace of olive oil factory. Bioresource Techn. 100:2375-2380.

34. ONWU, F.K.; OGAH, S.P. 2010. Studies on the effect of $\mathrm{pH}$ on the sorption of cadmium (II), nickel (II), lead (II) and chromium (VI) from aqueous solutions by african white star apple (Chrysophyllum albidium) shell. J. Biotechn. 9(42):7086-7093.

35. OSMAN, H.E; BADWY, R.K.; AHMAD, H.F. 2010. Usage of some agricultural by-products in theRemoval of some heavy metals from industrial wastewater. J. Phytol. 2(3):51-62.

36. PINZÓN-BEDOYA, M.L.; VERA-VILLAMIZAR, L.E. 2009. Modelamiento de la cinética de bioadsorción de $\mathrm{Cr}$ (III) usando cáscara de naranja. Dyna. 76(160):95106.

37. QAISER, S.; SALEEMI, A.; UMAR, M. 2009. Biosorption of lead(II) and chromium(VI) on groundnut hull: Equilibrium, kinetics and thermodynamics study. Electronic J. Biotechn.12(4):1-17.

38. RAFATULLAHA, M.; SULAIMANA, O.; HASHIMA, R.; AHMADB, A. 2009. Adsorption of copper (II), chromium (III), nickel (II) and lead (II) ions from aqueous solutions by meranti sawdust. J. Haz. Mat. 170:969-977.

39. RAO, R.; REHMAN, F. 2010. Adsorption studies on fruits of Gular (Ficus glomerata): Removal of $\mathrm{Cr}(\mathrm{VI})$ from synthetic wastewater. J. Haz. Mat. 181:405-412.

40. RAO-POPURI, S.; JAMMALA, A.; NAGA, S.R.; KACHIREDDY, V.; ABBURI, K. 2007. Biosorption of hexavalent chromium using tamarind (Tamarindus indica) fruit shell-a comparative study. Electronic J. Biotechn. 10(3):358-367.

41. RIAZA, M.; NADEEMA, R.; HANIFA, M.; ANSARIC, T.; REHMANA, K. 2009. Pb(II) biosorption from hazardous aqueous streams using Gossypium hirsutum (Cotton) waste biomass. J. Haz. Mat. 161(1):88-94. 
42. SAEED, A.; IQBAL, M.; HOLL, W. 2009. Kinetics, equilibrium and mechanism of $\mathrm{Cd}^{2+}$ removal from aqueous solution by mungbean husk. J. Haz. Mat. 168:1467-1475.

43. SHIH-WEI, L.A.; CHUN-I, L.; LI-HWA, W. 2011. Kinetic study on lead (II) ion removal by adsorption onto peanut hull ash. J. Taiwan Inst. Chem. Eng. 42:166172.

44. SINGH, K.; HASAN, S.; TALAT, M.; SINGH, V.; GANGWAR, S. 2009. Removal of $\mathrm{Cr}$ (VI) from aqueous solutions using wheat bran. J. Chem. Eng. 151:113121.

45. SRIVASTAVA, V.; MALL, I.; MISHRA, I. 2009. Competitive adsorption of cadmium(II) and nickel(II) metal ions from aqueous solution onto rice husk ash. Chem. Eng. Processing. 48:370-379.

46. TAPIA, N.J.; MUÑOZ, J.C.; TORRES, F.; YARANGO, A. 2003. Biosorción de plomo (II) por cascara de naranja, Citrus sinensis modificada. Rev. Per. Quim. Ing. Quim. 5(2):48-53.

47. TATY-COSTODES, V.C.; FAUDUET, H.; PORTE, C.; DELACROIX, A. 2003. Removal of Cd(II) and Pb(II) ions, fromaqueous solutions, by adsorption ontosawdust of Pinus sylvestris. J. Haz. Mat. 105:121-142.

48. THEVANNAN, A.; MUNGROO, R.; HUI, C. 2010. Biosorption of nickel with barley straw. Bioresource Techn. 101(6):1776-1780.
49. VENKATA, M.; SUBBAIAH, Y.; VIJAYA, N.; SIVA, K.; SUBBA, A.; KRISHNAIAH, A. 2009. Biosorption of nickel from aqueous solutions by Acacia leucocephala bark: Kinetics and equilibrium studies. Colloids and Surfaces B: Biointerfaces. 74(1):260-265.

50. WEBER, W.J.; MORRIS, J.C. 1963. Kinetics of adsorption on carbon from solution. J. San. Eng. Div. ASCE. 89:31-59.

51. WITEK-KROWIAK, A.; SZAFRAN, R.; MODELSKI, S. 2011. Biosorption of heavy metals from aqueous solutions onto peanut shell as a low-cost biosorbent. Desalination. 265:126-134.

52. YE, H.; ZHU, Q.; DU, D. 2010. Adsorptive removal of $\mathrm{Cd}(\mathrm{II})$ from aqueous solution using natural and modified rice husk. Bioresource Techn. 101:5175-5179.

53. ZABIHI, M.; HAGHIGHI, A.A.; AHMADPOUR, A. 2010. Studies on adsorption of mercury from aqueous solution on activated carbons prepared from walnut shell. J. Haz. Mat. 174:251-256.

54. ZAHEER, A.M.; RAMZAN, N.; NAVEED, S.Y.; FEROZE, N. 2010. Ni(II) removal by biosorption using Ficus religiosa (peepal) leaves. J. Chil. Chem. Soc. 55(1):8184.

Recibido: Octubre 23 de 2012

Aceptado: Octubre 10 de 2013

Como citar:

Quiñones, E.; Tejada, C.; Arcia, C.; Ruiz, V. 2013. Remoción de plomo y níquel en soluciones acuosas usando biomasas lignocelulósicas: una revisión. Rev. U.D.C.A Act. \& Div. Cient. 16(2): 479-489. 